Open J. Math. Sci., Vol. 2(2018), No. 1, pp. 228 - 239

Website: https://pisrt.org/psr-press/journals/oms/

ISSN: 2523-0212 (Online) 2616-4906 (Print)

http://dx.doi.org/10.30538/oms2018.0031

\title{
THE WEIGHTED SQUARE INTEGRAL INEQUALITIES FOR SMOOTH AND WEAK SUBSOLUTION OF FOURTH ORDER LAPLACE EQUATION
}

\author{
MUHAMMAD SHOAIB SALEEM ${ }^{1}$, J. PEČARIĆ, MOBEEN MUNIR, ASGHAR ALI, \\ MUHAMMAD SHAHID IQBAL TUBSSAM
}

\begin{abstract}
In this work we develop the weighted square integral estimates for the second derivatives of weak subsolution of forth order Laplace equation. It is natural generalization of inequalities develop for the Superharmonic functions in [1].

Mathematics Subject Classification: 60J20, 65L20.

Key words and phrases: fourth order Laplace equation; smooth subsolution; weak subsolution; adjoint operator.
\end{abstract}

\section{Introduction}

Among the most important of all partial differential equations are undoubtedly second order Laplace equation. Some of practical problems also covered by higher order Laplace equation. In [1], [2], [3], [4] and [5] the author develop the energy estimates for convex functions, 4-convex functions and also for super-harmonic functions. These estimates are very important in financial mathematics one can see [6], so it is also interesting to develop similar results for subsolution of fourth order Laplace equation. The fourth order Laplace equation with $n$ variables is given as

$$
\frac{\partial^{4} u(x)}{\partial x_{1}^{4}}+\frac{\partial^{4} u(x)}{\partial x_{2}^{4}}+\ldots+\frac{\partial^{4} u(x)}{\partial x_{n}^{4}}=0
$$

Received 12 March 2018. Revised 24 August 2018.

${ }^{1}$ Corresponding Author

(C) 2018 Muhammad Shoaib Saleem, J. Pečarić, Mubeen Munir, Asghar Ali, Muhammad Shahid Iqbal Tubssam. This is an open access article distributed under the Creative Commons Attribution License, which permits unrestricted use, distribution, and reproduction in any medium, provided the original work is properly cited. 
Let us denote

$$
\Delta^{4} \equiv \frac{\partial^{4}}{\partial x_{1}^{4}}+\frac{\partial^{4}}{\partial x_{2}^{4}}+\ldots+\frac{\partial^{4}}{\partial x_{n}^{4}}
$$

Then (1) becomes as

$$
\Delta^{4} u(x)=0
$$

The function $u(x) \in C^{4}(D)$ is called subsolution (suppersolution) of fourth order Laplace equation (2) if

$$
\Delta^{4} u(x) \geq(\leq) 0
$$

The bounded measurable function $u(x)$ is called week subsolution of (1) if $u(x)$ satisfy

$$
\int_{D} u(x) \Delta^{* 4} \psi(x) d x \geq 0
$$

It is trivial that $\Delta^{4}$ is self-adjoint operator i.e.

$$
\Delta^{4}=\Delta^{* 4}
$$

Through out the paper we will use the following notations.

$$
\begin{gathered}
\operatorname{grad} u(x)=\left(\frac{\partial u(x)}{\partial x_{1}}, \frac{\partial u(x)}{\partial x_{2}}, \ldots, \frac{\partial u(x)}{\partial x_{n}}\right) \\
\operatorname{grad}^{2} u(x)=\left(\frac{\partial^{2} u(x)}{\partial x_{1}^{2}}, \frac{\partial^{2} u(x)}{\partial x_{2}^{2}}, \ldots, \frac{\partial^{2} u(x)}{\partial x_{n}^{2}}\right)
\end{gathered}
$$

We will organize the paper in the following way in second section we will derive the energy estimate for the fourth order Laplace equation. Also we approximate the week subsolution by the smooth ones. In the last section we will derived similar estimate regarding week subsolution.

\section{The Weighted Energy Estimates for the Smooth Subsolution for the Fourth Order Laplace Equation.}

Lemma 2.1. [5] Assume $f(x) \in C^{4}(I)$ is the smooth and $h(x)$ is the smooth non-negative weight function having compact support. From the proof of Theorem 2.1 in [5] one can derive the identity in Lemma 2.1 as:

$$
\begin{aligned}
\int_{I}\left(f^{\prime \prime}(x)\right)^{2} h(x) d x & =\int_{I} f(x) f^{(i v)}(x) h(x) d x-2 \int_{I} f(x) f^{\prime \prime}(x) h^{\prime \prime}(x) d x \\
& +\frac{1}{2} \int_{I} f^{2}(x) h^{(i v)}(x) d x
\end{aligned}
$$


Theorem 2.2. Let $u_{i}(x) \in C^{4}(D), i=1,2$ be the smooth subsolutions of (2) over the domain $D \subseteq \mathbb{R}^{n}$ and also $\frac{\partial^{2} u_{i}}{\partial x_{j}^{2}} \geq 0 \forall j=1,2, \ldots, n$, then we have the following energy estimate for the difference of the functions

$$
\begin{aligned}
& \int_{D}\left|\operatorname{grad}^{2}\left(u_{2}(x)-u_{1}(x)\right)\right|^{2} h(x) d x \\
& \leq \int_{D}\left[\frac{1}{2}\left(u_{2}(x)-u_{1}(x)\right)^{2}\right. \\
& \left.-\sup _{x \in D}\left|\left(u_{2}(x)-u_{1}(x)\right)\right|\left(u_{2}(x)+u_{1}(x)\right)\right] \sum_{j=1}^{n} \frac{\partial^{4} h(x)}{\partial x_{j}^{4}} d x
\end{aligned}
$$

where $h(x)$ is the non-negative smooth weight function satisfying

$$
\left\{\begin{array}{l}
h(x)=\frac{\partial h(x)}{\partial x_{j}}=\frac{\partial^{2} h(x)}{\partial x_{j}^{2}}=\frac{\partial^{3} h(x)}{\partial x_{j}^{3}}=0 \quad j=1, \ldots, n \forall x \in \partial D \\
\frac{\partial^{2} h(x)}{\partial x_{j}^{2}} \leq 0 \quad j=1, \ldots, n \text { for } x \in D .
\end{array}\right.
$$

Proof. Let

$$
u(x)=u_{2}(x)-u_{1}(x)
$$

Take

$$
\begin{gathered}
\int_{D}\left|\operatorname{grad}^{2} u(x)\right|^{2} h(x) d x=\int_{D}\left[\left(\frac{\partial^{2} u(x)}{\partial x_{1}^{2}}\right)^{2}+\left(\frac{\partial^{2} u(x)}{\partial x_{2}^{2}}\right)^{2}\right. \\
\left.+\ldots+\left(\frac{\partial^{2} u(x)}{\partial x_{n}^{2}}\right)^{2}\right] h(x) d x \\
=\int_{D}\left(\frac{\partial^{2} u(x)}{\partial x_{1}^{2}}\right)^{2} h(x) d x+\int_{D}\left(\frac{\partial^{2} u(x)}{\partial x_{2}^{2}}\right)^{2} h(x) d x+\ldots+ \\
\int_{D}\left(\frac{\partial^{2} u(x)}{\partial x_{n}^{2}}\right)^{2} h(x) d x
\end{gathered}
$$

By Lemma 2.1, the above may write as

$$
\begin{gathered}
=\sum_{j=1}^{n}\left(\int_{D} u(x) \frac{\partial^{4} u(x)}{\partial x_{j}^{4}} h(x) d x-2 \int_{D} u(x) \frac{\partial^{2} u(x)}{\partial x_{j}^{2}} \frac{\partial^{2} h(x)}{\partial x_{j}^{2}} d x+\frac{1}{2} \int_{D} u^{2}(x) \frac{\partial^{4} h(x)}{\partial x_{j}^{4}} d x\right) \\
\leq \int_{D}|u(x)|\left|\sum_{j=1}^{n} \frac{\partial^{4} u(x)}{\partial x_{j}^{4}}\right| h(x) d x+2 \int_{D}|u(x)|\left|\sum_{j=1}^{n} \frac{\partial^{2} u(x)}{\partial x_{j}^{2}} \frac{\partial^{2} h(x)}{\partial x_{j}^{2}}\right| d x \\
+\frac{1}{2} \int_{D} u^{2}(x) \sum_{j=1}^{n} \frac{\partial^{4} h(x)}{\partial x_{j}^{4}} d x
\end{gathered}
$$




$$
\begin{gathered}
\leq \sup _{x \in D}|u(x)| \int_{D}\left|\sum_{j=1}^{n} \frac{\partial^{4} u(x)}{\partial x_{j}^{4}}\right| h(x) d x+2 \sup _{x \in D}|u(x)| \int_{D}\left|\sum_{j=1}^{n} \frac{\partial^{2} u(x)}{\partial x_{j}^{2}} \frac{\partial^{2} h(x)}{\partial x_{j}^{2}}\right| d x \\
+\frac{1}{2} \int_{D} u^{2}(x) \sum_{j=1}^{n} \frac{\partial^{4} h(x)}{\partial x_{j}^{4}} d x
\end{gathered}
$$

Now replacing $u(x)=u_{2}(x)-u_{1}(x)$ we obtain,

$$
\begin{gathered}
\int_{D}\left|\operatorname{grad}^{2}\left(u_{2}(x)-u_{1}(x)\right)\right|^{2} h(x) d x \\
\leq \sup _{x \in D}\left|\left(u_{2}(x)-u_{1}(x)\right)\right| \int_{D}\left|\sum_{j=1}^{n} \frac{\partial^{4}}{\partial x_{j}^{4}}\left(u_{2}(x)-u_{1}(x)\right)\right| h(x) d x \\
+2 \sup _{x \in D}\left|\left(u_{2}(x)-u_{1}(x)\right)\right| \int_{D}\left|\sum_{j=1}^{n} \frac{\partial^{2}}{\partial x_{j}^{2}}\left(u_{2}(x)-u_{1}(x)\right) \frac{\partial^{2} h(x)}{\partial x_{j}^{2}}\right| d x \\
+\frac{1}{2} \int_{D}\left(u_{2}(x)-u_{1}(x)\right)^{2} \sum_{j=1}^{n} \frac{\partial^{4} h(x)}{\partial x_{j}^{4}} d x \\
\leq \sup _{x \in D}\left|u_{2}(x)-u_{1}(x)\right| \sum_{j=1}^{n} \int_{D}\left(\frac{\partial^{4} u_{2}(x)}{\partial x_{j}^{4}}+\frac{\partial^{4} u_{1}(x)}{\partial x_{j}^{4}}\right) h(x) d x \\
-2 \sup _{x \in D}\left|u_{2}(x)-u_{1}(x)\right| \sum_{j=1}^{n} \int_{D}\left(\frac{\partial^{2} u_{2}(x)}{\partial x_{j}^{2}}+\frac{\partial^{2} u_{1}(x)}{\partial x_{j}^{2}}\right) \frac{\partial^{2} h(x)}{\partial x_{j}^{2}} d x \\
+\frac{1}{2} \int_{D}\left(u_{2}(x)-u_{1}(x)\right)^{2} \sum_{j=1}^{n} \frac{\partial^{4} h(x)}{\partial x_{j}^{4}} d x
\end{gathered}
$$

Again using the definition of weight function and integration by parts formula, we obtain

$$
\int_{D} \sum_{j=1}^{n} \frac{\partial^{4} u_{i}(x)}{\partial x_{j}^{4}} h(x) d x=\int_{D} u_{i}(x) \sum_{j=1}^{n} \frac{\partial^{4} h(x)}{\partial x_{j}^{4}} d x \quad i=1,2
$$

and

$$
\int_{D} \sum_{j=1}^{n} \frac{\partial^{2} u_{i}(x)}{\partial x_{j}^{2}} \frac{\partial^{2} h(x)}{\partial x_{j}^{2}} d x=\int_{D} u_{i}(x) \sum_{j=1}^{n} \frac{\partial^{4} h(x)}{\partial x_{j}^{4}} d x \quad i=1,2
$$

the above (10) becomes

$$
\int_{D}\left|\operatorname{grad}^{2}\left(u_{2}(x)-u_{1}(x)\right)\right|^{2} h(x) d x \leq
$$




$$
\int_{D}\left(\frac{1}{2}\left(u_{2}(x)-u_{1}(x)\right)^{2}-\sup _{x \in D}\left|u_{2}(x)-u_{1}(x)\right|\left(u_{2}(x)+u_{1}(x)\right)\right) \sum_{j=1}^{n} \frac{\partial^{4} h(x)}{\partial x_{j}^{4}} d x
$$

Remark 2.1. Taking the supremum norm on above inequality we obtained

$$
\begin{aligned}
& \int_{D}\left|\operatorname{grad}^{2}\left(u_{2}(x)-u_{1}(x)\right)\right|^{2} h(x) d x \\
& \leq\left[\frac{1}{2}\left\|u_{2}(x)-u_{1}(x)\right\|_{L^{\infty}}^{2}+\left\|u_{2}(x)-u_{1}(x)\right\|_{L^{\infty}}\right. \\
& \left.\times\left(\left\|u_{1}(x)\right\|_{L^{\infty}}+\left\|u_{2}(x)\right\|_{L^{\infty}}\right)\right] \\
& \times \int_{D} \Delta^{4} h(x) d x .
\end{aligned}
$$

Remark 2.2. The above estimates over domain $D \in \mathbb{R}^{n}$ also holds for arbitrary ball $B\left(x_{o}, r\right)$ with center $x_{o}$ and radius $r$.

From now we use ball $B\left(x_{o}, r\right)$ as domain in $\mathbb{R}^{n}$.

\section{The Weighted Energy Estimates for the Week Subsolution of Fourth Order Laplace Equation.}

The continuous function $u(x)$ is said to be week subsolution of (2) if

$$
\int_{B} u(x) \Delta^{4} \psi(x) d x \geq 0
$$

where

$$
\psi \in C_{c}^{\infty}(B) .
$$

Now we will approximate the week subsolution of (2) by the smooth ones. For this we will use the classical mollification. Define

$$
\eta(x)= \begin{cases}c \exp \left(\frac{1}{|x|^{2}-1}\right) & \text { if }|x|<1 \\ 0 & \text { if }|x| \geq 1\end{cases}
$$

where $x \in \mathbb{R}^{n}$ and $c>0$ is such that

$$
\int_{\mathbb{R}^{n}} \eta(x) d x=1
$$

Now we define

$$
u_{\epsilon}(x)=\epsilon^{-n} \int_{B} \eta\left(\frac{x-y}{\epsilon}\right) u(y) d y
$$

Let us denote

$$
\eta_{\epsilon}(x-y)=\epsilon^{-n} \eta\left(\frac{x-y}{\epsilon}\right)
$$


By the definition $\eta_{\epsilon}(x-y)$, It is trivial that

$$
\frac{\partial^{4} \eta_{\epsilon}(x-y)}{\partial x_{i}^{4}}=\frac{\partial^{4} \eta_{\epsilon}(x-y)}{\partial y_{i}^{4}} \quad \forall i=1,2, \ldots, n
$$

so,

$$
\Delta_{x}^{4} u_{\epsilon}(x)=\epsilon^{-n} \int_{B} u(y) \Delta_{y}^{4} \eta_{\epsilon}(x-y) d y
$$

where $\Delta_{x}^{4}$ and $\Delta_{y}^{4}$ are the fourth order Laplace operator w.r.t $x$ and $y$ respectively.

Let the ball $B_{k}=B\left(x_{o}, r_{k}\right)$ with $r_{k}=\frac{k+1}{k+2} r \quad, k=1,2$, .

The next theorem tells about the existence of smooth approximation.

Theorem 3.1. Let $u(x)$ be the continuous week subsolution of (2) and convex over the ball $B$. Then for any $k=1,2,3, \ldots$, there exists $\hat{\epsilon}>0$ such that $0<$ $\epsilon<\hat{\epsilon}$ each function $u_{\epsilon}(x)$ is smooth convex over the ball $B_{k}$ and also

$$
\Delta^{4} u_{\epsilon}(x) \geq 0 \text { if } x \in B_{k} .
$$

Proof. For fixed $k=1,2, \ldots$,

Let

$$
\hat{\epsilon}=\frac{r}{2(k+2)}
$$

By the definition, it is trivial that $u_{\epsilon}(x)$ is infinitely differentiable and also from [7] $u_{\epsilon}(x)$ is smooth convex for each of its arguments.

we next claim that $\eta_{\epsilon}(x-y)$ has compact support in the ball $B$.

Take another ball $\hat{B}_{k}$ in the following way

$$
\begin{gathered}
\hat{B}_{k}=B\left(x_{0}, \frac{2 k+3}{2 k+4} r\right) \\
\text { If } y \notin \hat{B}_{k} \text { then } \\
|y-x|>\left|\frac{2 k+3}{2 k+4}-\frac{2 k+2}{2 k+4}\right| r=\frac{1}{2(k+2)} r>\epsilon \\
\Rightarrow \quad \eta_{\epsilon}(x-y)=0
\end{gathered}
$$

Hence $\eta_{\epsilon}(x-y)$ has compact support.

Hence by the definition of week subsolution and also using (22), we get

$$
\int_{B} u(y) \Delta_{y}^{4} \eta_{\epsilon}(x-y) d y \geq 0 .
$$


We will defined $h_{k}(x)$ as:

$$
\left\{\begin{array}{l}
h_{k}(x)>0 \quad \text { if } \quad x \in B_{k}\left(x_{0}, r_{k}\right) \\
h_{k}(x)=0 \quad \text { if } \quad x \in \partial B_{k}\left(x_{0}, r_{k}\right)
\end{array}\right.
$$

where $r_{k}=\frac{k+1}{k+2} r$

Theorem 3.2. Let $u(x)$ be the continuous week subsolution of (2) and convex over the ball $B$ then it possesses the following weak partial derivatives $\frac{\partial^{2} u(x)}{\partial x_{i}^{2}}, i=$ $1,2, \ldots, n$ over the ball $B$.

Proof. For the existence of first derivative $\frac{\partial u(x)}{\partial x_{i}} i=1,2, \ldots, n$ one can see [4]. Let us suppose the mollification $u_{\epsilon}(x)$ defined in (15) for the week subsolution of fourth order Laplace equation $u(x)$.

For the continuous function $u(x)$, the ball $B$, it is well-known fact that on compact set $\subseteq B$ we have the following uniform-convergence

$$
\sup _{k}\left|u_{\epsilon}(x)-u(x)\right| \underset{\epsilon \rightarrow 0}{\longrightarrow} 0 .
$$

Let us denote $u_{\epsilon}(x)$ by $u_{m}(x)$ for $\epsilon=\frac{1}{m}, m=1,2, \ldots$ so above becomes

$$
\sup _{k}\left|u_{m}(x)-u(x)\right| \underset{m \rightarrow \infty}{\longrightarrow} 0
$$

The ball $B_{k}$ are compactly contained in the original ball $B$

From the theorem 3.1 , we know that for any $\mathrm{k}=1,2, \ldots$, there exists $m_{k}$ such that $u_{m}(x)$ is smooth subsolution of $(2)$

Take the ball $B_{k+l}$ and write the inequality (11) for

$$
\begin{aligned}
& u_{1}(x)=u_{m}(x) \text { and } u_{2}(x)=u_{p}(x) \\
& \int_{B_{k+l}}\left|g_{r a d} u_{p}-g_{r a d} u_{m}\right|^{2} h_{k+l} d x \\
& \leq\left[\frac{1}{2}\left\|u_{p}-u_{m}\right\|_{L^{\infty}}^{2}+\left\|u_{p}-u_{m}\right\|_{L^{\infty}}\left(\left\|u_{p}\right\|_{L^{\infty}}+\left\|u_{m}\right\|_{L^{\infty}}\right)\right] \\
& \times \int_{B_{k+l}}\left|\Delta^{4} h_{k+l}\right| d x
\end{aligned}
$$

Let us denote

$$
\begin{gathered}
\alpha_{k+l}=\int_{B_{k+l}}\left|\Delta^{4} h_{k+l}\right| d x, \\
\hat{\alpha}=\inf _{x \in B_{k+l}} h_{k+l},
\end{gathered}
$$

then (25) becomes

$$
\hat{\alpha} \int_{B_{k+l}}\left|\operatorname{grad}^{2} u_{p}-\operatorname{grad}^{2} u_{m}\right|^{2} d x
$$




$$
\begin{aligned}
& \leq \alpha_{k+l}\left[\frac{1}{2}\left\|u_{p}-u_{m}\right\|_{L^{\infty}}^{2}+\left\|u_{p}-u_{m}\right\|_{L^{\infty}}\right. \\
& \left.\times\left(\left\|u_{p}\right\|_{L^{\infty}}+\left\|u_{m}\right\|_{L^{\infty}}\right)\right]
\end{aligned}
$$

where $\hat{\alpha} \neq 0$. Writing the left hand integral for the smaller ball $B_{k}$, we have

$$
\begin{aligned}
& \hat{\alpha} \int_{B_{k}}\left|\operatorname{grad}^{2} u_{p}-\operatorname{grad}^{2} u_{m}\right|^{2} d x \\
& \leq \alpha_{k+l}\left[\frac{1}{2}\left\|u_{p}-u_{m}\right\|_{L^{\infty}}^{2}+\left\|u_{p}-u_{m}\right\|_{L^{\infty}}\right. \\
& \left.\times\left(\left\|u_{p}\right\|_{L^{\infty}}+\left\|u_{m}\right\|_{L^{\infty}}\right)\right]
\end{aligned}
$$

From (24), we have

$$
\left\|u_{p}-u_{m}\right\|_{L_{\left(B_{k+l}\right)}^{\infty}} \longrightarrow 0 \text { as } \quad m, p \longrightarrow \infty
$$

so (27) becomes.

$$
\lim _{m, p \rightarrow \infty} \sum_{i=1}^{n} \int_{B_{k}}\left(\frac{\partial^{2} u_{p}}{\partial x_{i}^{2}}-\frac{\partial^{2} u_{m}}{\partial x_{i}^{2}}\right) d x=0
$$

The completeness of $L^{2}\left(B_{k}\right)$ ensure the convergence of above sequence. So there exist a class of measurable functions $v_{k, i}(x) \in L^{2}\left(B_{k}\right)$ such that

$$
\sum_{i=1}^{n} \int_{B_{k}}\left(\frac{\partial^{2} u_{m}}{\partial x_{i}^{2}}-v_{k, i}\right)^{2} d x \underset{m \rightarrow \infty}{\longrightarrow} 0, k=1,2, \ldots
$$

we extend $v_{k, i}(x)$ trivially outside the ball $B_{k}$ by 0 .

Let us denote

$$
v_{i}(x)=\lim _{k \rightarrow \infty} \sup _{x \in D} v_{k, i}, \quad i=1,2, \ldots, n
$$

It can be checked easily that

$$
v_{i}(x)=v_{k, i}
$$

for the ball $B_{k}$.

Next we claim that $v_{i}(x)$ represent the weak second order partial derivative $\frac{\partial^{2} u(x)}{\partial x_{i}^{2}}$ of $u(x)$.

Take $\psi(x)$ an arbitrary function having compact support in B.

Then suppose $\psi(x) \subset B_{k}$ from some $\mathrm{k}$.

We have

$$
\int_{B} \frac{\partial^{2} u_{m}}{\partial x_{i}^{2}} \psi d x=\int_{B} u_{m} \frac{\partial^{2} \psi}{\partial x_{i}^{2}} d x
$$


for the integers $m \geq m_{k}$.

But we have the following convergence

$$
\left|u_{m}(x)-u(x)\right| \underset{m \rightarrow \infty}{\longrightarrow} 0 \text { on } B_{k}
$$

and

$$
\left\|\frac{\partial^{2} u_{m}}{\partial x_{i}^{2}}-v_{i}(x)\right\|_{L^{2}\left(B_{k}\right)} \underset{m \rightarrow \infty}{\longrightarrow} 0
$$

using these Limits, the above becomes.

$$
\int_{B_{k}} v_{i}(x) \psi(x) d x=\int_{B_{k}} u(x) \frac{\partial^{2} \psi}{\partial x_{i}^{2}} d x .
$$

This shows that $v_{i}(x) i=1,2, \ldots, n$ are the weak partial derivative of $u(x)$. Rewriting the inequality (11) for the functions $u_{1}(x)=0$ and $u_{2}(x)=u_{m}(x)$ for $m \geq m_{k+l}$ over the ball $B_{k+l}$, we get

$$
\int_{B_{k+l}}\left|\operatorname{grad}^{2} u_{m}(x)\right|^{2} h_{k+l}(x) d x \leq \frac{3}{2} \alpha_{k+l}\left\|u_{m}(x)\right\|_{L^{\infty}\left(B_{k+l}\right)}^{2}
$$

Taking limit $m \rightarrow \infty$, the above becomes

$$
\int_{B_{k+l}}\left|\operatorname{grad}^{2} u_{m}(x)\right|^{2} h_{k+l}(x) d x \leq \frac{3}{2} \alpha_{k+l}\|u(x)\|_{L^{\infty}\left(B_{k+l}\right)}^{2}
$$

Now restricting the left hand side for the smaller ball $B_{k}$, we have

$$
\int_{B_{k}}\left|\operatorname{grad}^{2} u_{m}(x)\right|^{2} h_{k+l}(x) d x \leq \frac{3}{2} \alpha_{k+l}\|u(x)\|_{L^{\infty}\left(B_{k+l}\right)}^{2}
$$

Now making limit as $m \rightarrow \infty$, we obtain

$$
\int_{B_{k}}\left|\operatorname{grad}^{2} u(x)\right|^{2} h(x) d x \leq \frac{3}{2} \alpha_{\infty}\|u(x)\|_{L^{\infty}(B)}^{2}<\infty .
$$

The left hand side of above increases as $k$ increases and also bounded, so it will have finite limit, i.e

$$
\int_{B}\left|\operatorname{grad}^{2} u(x)\right|^{2} h(x) d x \leq \frac{3}{2} \alpha_{\infty}\|u(x)\|_{L^{\infty}(B)}^{2}<\infty .
$$

which completes the proof.

Now we prove the inequality for the weak subsolution of fourth order Laplace equation.

Theorem 3.3. Let $u_{i}(x), i=1,2$ be the continuous weak subsolution of (2), and also it satisfies

$$
\frac{\partial^{2} u(x)}{\partial x_{j}^{2}} \geq 0 \quad \forall j=1,2, \ldots, n
$$


then the following is valid

$$
\begin{aligned}
\int_{B}\left|\operatorname{grad}^{2} u_{2}(x)-\operatorname{grad}^{2} u_{1}(x)\right|^{2} h(x) d x \leq & \leq\left[\frac{1}{2}\left\|u_{2}(x)-u_{1}(x)\right\|_{L^{\infty}(B)}^{2}\right. \\
& +\left\|\left(u_{2}(x)-u_{1}(x)\right)\right\|_{L^{\infty}(B)} \\
& \left.\times\left(\left\|u_{1}(x)\right\|_{L^{\infty}(B)}+\left\|u_{2}(x)\right\|_{L^{\infty}(B)}\right)\right] \\
& \times \int_{B}\left|\Delta^{4} h(x)\right| d x
\end{aligned}
$$

where $h(x)$ is the weight function satisfying (7).

Proof. Take mollification $u_{m, i}(x)$, for $i=1,2$ of the continuous weak subsolution $u_{i}(x)$, for $i=1,2$ respectively.

Since for the ball $B_{k+l}$, there exists integer $m_{k+l}$, such that each function $u_{m, i}$, for $i=1,2$ is the smooth subsolution in the ball $B_{k+l}$, if $m>m_{k+l}$. Also we have the following convergence

$$
\left\|u_{m, i}(x)-u_{i}(x)\right\|_{L^{\infty}\left(B_{k+l}\right)} \underset{m \rightarrow \infty}{\longrightarrow} 0 \quad i=1,2 .
$$

Now writing the inequalities the function $u_{m, i}(x), \mathrm{i}=1,2$ and the ball $B_{k+l}$, we get

$$
\begin{aligned}
& \int_{B_{k+l}}\left|\operatorname{grad}^{2} u_{m, 2}(x)-\operatorname{grad}^{2} u_{m, 1}(x)\right|^{2} h_{k+l}(x) d x \\
& \leq \alpha_{k+l}\left[\frac{1}{2}\left\|u_{m, 2}(x)-u_{m, 1}(x)\right\|_{L^{\infty}\left(B_{k+l}\right)}^{2}+\| u_{m, 2}(x)-u_{m, 1}(x)\right) \|_{L^{\infty}\left(B_{k+l}\right)} \\
& \left.\times\left(\left\|u_{m, 1}(x)\right\|_{L^{\infty}\left(B_{k+l}\right)}+\left\|u_{m, 2}(x)\right\|_{L^{\infty}\left(B_{k+l}\right)}\right)\right]
\end{aligned}
$$

Taking Limit as $m \rightarrow \infty$, the latter inequality (30) becomes

$$
\begin{aligned}
& \int_{B_{k+l}}\left|\operatorname{grad}^{2} u_{2}(x)-\operatorname{grad}^{2} u_{1}(x)\right|^{2} h_{k+l}(x) d x \\
& \leq \alpha_{k+l}\left[\frac{1}{2}\left\|u_{2}(x)-u_{1}(x)\right\|_{L^{\infty}\left(B_{k+l}\right)}^{2}+\left\|u_{2}(x)-u_{1}(x)\right\|_{L^{\infty}\left(B_{k+l}\right)}\right. \\
& \left.\times\left(\left\|u_{1}(x)\right\|_{L^{\infty}\left(B_{k+l}\right)}+\left\|u_{2}(x)\right\|_{L^{\infty}\left(B_{k+l}\right)}\right)\right]
\end{aligned}
$$

Again writing the above inequality, the left hand side for smaller ball $B_{k}$ and also taking Limit $l \rightarrow \infty$, we get, we finally obtain

$$
\int_{B_{k}}\left|\operatorname{grad}^{2} u_{2}(x)-\operatorname{grad}^{2} u_{1}(x)\right|^{2} h(x) d x
$$




$$
\begin{aligned}
& \leq \alpha_{\infty}\left[\frac{1}{2}\left\|u_{2}(x)-u_{1}(x)\right\|_{L^{\infty}(B)}^{2}+\left\|u_{2}(x)-u_{1}(x)\right\|_{L^{\infty}(B)}\right. \\
& \left.\times\left(\left\|u_{1}(x)\right\|_{L^{\infty}(B)}+\left\|u_{2}(x)\right\|_{L^{\infty}(B)}\right)\right]
\end{aligned}
$$

The above inequalities holds for all $B_{k}, \mathrm{k}=1,2,3, \ldots, \mathrm{n}$. So also true for ball B. Which completes the proof.

\section{Conflict of Interests}

The authors hereby declare that there is no conflict of interests regarding the publication of this paper.

\section{REFERENCES}

1. Pečarić, J., Saleem, M. S., Rehman, H. U., Nizami, A. M., \& Hussain, A. (2015). Reverse Poincar-type inequalities for the difference of superharmonic functions. Journal of Inequalities and Applications, 2015(1), 400. https://doi.org/10.1186/s13660-015-0916-9

2. Hussain, S., Pečarić, J., \& Shashiashvili, M. (2008). The weighted square integral inequalities for the first derivative of the function of a real variable. Journal of Inequalities and Applications, 2008(1), 343024. https://doi.org/10.1155/2008/343024

3. Shashiashvili, K., \& Shashiashvili, M. (2005). Estimation of the derivative of the convex function by means of its uniform approximation. Journal of Inequalities in Pure and Applied Mathematics, 6(4), 1-10.

4. Saleem, M. S., \& Shashiashvili, M. (2010). The weighted reverse Poincar inequality for the difference of two weak subsolutions. Bull. Georg. Natl. Acad. Sci, 4(3), 24-28.

5. Saleem, M. S., Pečarić, J., Ahmad, I., \& Ahmad, N. (2018). The Weighted Integral Inequality for the Second Derivative of 4-Convex Function. Journal of Mathematical Inequalities, accepted for publication.

6. Hussain, S., \& Shashiashvili, M. (2010). Discrete time hedging of the American option. Mathematical Finance: An International Journal of Mathematics, Statistics and Financial Economics, 20(4), 647-670. https://doi.org/10.1111/j.1467-9965.2010.00415.x

7. Evans, L. C. (1998). Partial differential equations (Graduate studies in Mathematics, v. 19, American Mathematical Society, Providence, RI).

\section{Muhammad Shoaib Saleem}

Department of Mathematics, University of Okara, Okara, Pakistan.

e-mail: shaby455@yahoo.com

\section{J. Pečarić}

Faculty of Textile Technology, University of Zagreb, 10000, Zagreb Croatia.

e-mail: pecaric@element.hr

\section{Mobeen Munir}

Department of Mathematics, Division of Science and Technology, University of Education, Lahore 54000, Pakistan.

e-mail: mmunir@ue.edu.pk

\section{Asghar Ali}

Department of Mathematics and Statistics, The University of Lahore, Lahore 54000, Pakistan.

e-mail: asghar.toor@gmal.com 
Muhammad Shahid Iqbal Tubssam

Department of Mathematics and Statistics, The University of Lahore, Lahore 54000, Pakistan

e-mail: sindhu.shahid56@gmail.com 